\title{
Hip Arthroscopy for Femoroacetabular Impingement Syndrome Results in 2 Recovery Patterns Based on Preoperative Pain and on Arthritis: Improvers and Non-improvers
}

\author{
Maarten A. Röling, M.D., Brechtje Hesseling, M.Sc., Nina M. C. Mathijssen, Ph.D., and \\ Rolf M. Bloem, Ph.D.
}

Purpose: To analyze whether subgroups of patients could be distinguished with different functional recovery trajectories after hip arthroscopy for femoroacetabular impingement (FAI) syndrome and to identify differences between those subgroups using data from our prospective cohort registration. Methods: We retrospectively reviewed the prospectively registered data of patients who had undergone hip arthroscopy for FAI syndrome in our clinic from 2015 through 2018. Latent class growth modeling and growth mixture modeling were used to identify and classify groups of patients according to the trajectory of functional recovery using the Hip Outcome Score-Activities of Daily Life (HOS-ADL). We used univariable analysis and descriptive statistics to explore whether differences in group membership could be identified. Results: A total of 100 patients were analyzed. Growth mixture modeling identified 2 main types of recovery pattern after surgery: patients in whom the HOS-ADL improved significantly after surgery to 90, whom we called the "improvers" (with fast initial improvement within 3 months that is maintained during follow-up), and patients who did not significantly benefit from surgery (with only mild improvement in the HOS-ADL at 3 months and no further change during follow-up), whom we called the "non-improvers." Univariable analysis and comparison of differences between subgroups showed higher preoperative visual analog scale scores for pain and more intraoperative arthrosis of the femoral head for the non-improvers. Conclusions: We identified 2 main types of recovery pattern after arthroscopic treatment of FAI syndrome: improvers and non-improvers. Both groups recover in a different manner postoperatively. Preoperative pain and intraoperative arthrosis of the femoral head differed in the non-improvers compared with the improvers. Level of evidence: Level III, retrospective cohort study.

A rthroscopic surgery to resolve cam- and pincertype morphology causing femoroacetabular impingement (FAI) syndrome has been recognized as an effective treatment with good to excellent results. ${ }^{1}$ Most patients recover well in terms of regaining the

From the Department of Orthopaedic Surgery, Gelre Hospital Apeldoorn, Apeldoorn, The Netherlands (M.A.R.); Department of Orthopaedic Surgery, Reinier de Graaf Gasthuis, Delft, The Netherlands (B.H., N.M.C.M., R.M.B.).

The authors report no conflicts of interest in the authorship and publication of this article. Full ICMJE author disclosure forms are available for this article online, as supplementary material.

Received July 23, 2020; accepted June 30, 2021.

Address correspondence to Maarten A. Röling, M.D., Department of Orthopaedic Surgery, Gelre Hospital Apeldoorn, Albert Schweitzerlaan 31, 7334 DZ Apeldoorn, The Netherlands.E-mail:m.roling@gelre.nl

(C) 2021 THE AUTHORS. Published by Elsevier Inc. on behalf of the Arthroscopy Association of North America. This is an open access article under the CC BY-NC-ND license (http://creativecommons.org/licenses/by-nc-nd/4.0/). 2666-061X/201200

https://doi.org/10.1016/j.asmr.2021.06.014 prior sports level, pain reduction, and functional outcomes. $^{2-5}$ As with any operative procedure, multiple studies have emphasized proper patient selection for achieving good operative results because not all patients experience optimal recovery, which can lead to poor outcomes or even revision of the procedure. ${ }^{6-8}$ It is important to better understand differences between patients in how they respond to and recover from hip arthroscopy to further improve functional outcomes. Statistical techniques that are increasingly being used for this matter are latent class growth modeling (LCGM) and growth mixture modeling (GMM). ${ }^{9}$ These are an extension of latent growth curve modeling, or the mathematical equivalent, which is a mixed or multilevel model. ${ }^{10} \mathrm{~A}$ mixed model applied to longitudinal data allows for estimating the degree of heterogeneity between patients in recovery trajectories by estimating the random slope variance. ${ }^{11}$ This is a suitable method to investigate heterogeneity in a 
patient cohort in change patterns for outcome results and performs subgrouping based on pattern recognition with high accuracy. This statistical method enables us to identify different types of recovery patterns in subgroups in a cohort of patients. ${ }^{12-14}$ Previous and recent applications have, for instance, analyzed subgroups of patients according to their hip function trajectory during the first 6 weeks after total hip arthroplasty. ${ }^{15}$ Other applications have addressed the wide variety in patients' responses to total knee arthroplasty or cardiac rehabilitation. ${ }^{16,17}$ To our knowledge, no other study has used such a model to examine changes in patientreported outcomes (PROs) after hip arthroscopy for FAI syndrome.

We applied LCGM and GMM to prospectively collected data of patients operated on for FAI syndrome using hip arthroscopy to determine subgroups of patients according to their functional outcome results, as measured with the Hip Outcome Score-Activities of Daily Life (HOS-ADL). Furthermore, we set out to determine associations of group membership with preoperative and intraoperative parameters.

The purposes of this study were to analyze whether subgroups of patients could be distinguished with different functional recovery trajectories after hip arthroscopy for FAI syndrome and to identify differences between those subgroups using data from our prospective cohort registration. Our hypotheses were that at least 2 subgroups with different functional recovery trajectories could be identified and that several differences could be identified by comparing groups.

\section{Methods}

\section{Data Collection}

We retrospectively reviewed prospectively registered data of patients who had undergone hip arthroscopy for FAI syndrome in our clinic from 2015 through 2018. Loss to follow-up was reported. Patients were selected to undergo elective hip arthroscopy for FAI syndrome according to our local protocol. The inclusion criteria in this protocol are as follows: diagnosis of FAI syndrome (according to the Warwick Agreement), ${ }^{5}$ that is, positive clinical assessment findings with positive test results for FAI, ${ }^{18}$ as well as radiologic assessment findings conclusive for FAI (with cam and/or pincer morphology on radiography and/or a labral tear on magnetic resonance imaging); age 18 to 65 years; conservative management initially (with strengthening physiotherapy for at least 3 months, lifestyle changes, and nonsteroidal anti-inflammatory drugs); suitability for surgery (after consultation with the anesthesiologist regarding any contraindications for surgery); and willingness of patient to participate and sign the informed consent form. The exclusion criteria were signs of progressive osteoarthritis (Tönnis grade $>2$ ), revision hip arthroscopy, or metastatic pathologic disease.

The study protocol (No. METCZWK 12-083) was assessed by our regional medical ethical committee, which decided that the study did not fall under the scope of the Medical Research Involving Human Subject Act because of the minimal burden on patients in comparison to regular care. The trial was registered in the Netherlands Trial Register (NTR6792). Arthroscopic surgery was performed by an experience orthopaedic surgeon (RMB) in a large educational hospital in Delft, the Netherlands.

After informed consent was obtained, all patients were asked to fill in PRO questionnaires preoperatively and postoperatively at 3, 12, and 24 months. The patient assessment did not differ from normal clinical practice according to our local protocol.

Patient data were included with a postoperative followup and data registration period of 24 months. Obtained data comprised patient characteristics (age, sex, body mass index [BMI], American Society of Anesthesiologists score, length of complaints in years, preoperative diagnoses [cam morphology, pincer morphology, or labral tear], and signs of osteoarthritis [Tönnis grading]); preoperative range of motion of the hip joint; intraoperatively identified pathologies of the central and peripheral compartments; and several preoperative and postoperative questionnaires. All patients were physically examined for complications postoperatively, as well as at 6 weeks, 3 months, and 12 months. The PRO questionnaires included a visual analog scale (VAS) for pain, the HOS-ADL, the Hip Outcome Score (HOS)-Sport, and the EuroQoL-5D (EQ5D-5L) for mobility for preoperative and postoperative assessment, as well as the 4-Dimensional Symptom Questionnaire (4-DSQ) at the postoperative assessment.

The VAS pain score uses a VAS with a 10-cm-wide range; the score ranges from 0 to 100 . On the VAS, patients point out the amount of pain they have, with 0 indicating no pain and 100 indicating the worst possible pain.

The EQ5D-5L is a standardized instrument developed by the EuroQol Group ${ }^{19}$ as a measure of health-related quality of life that can be used in a wide range of health conditions. It contains 5 domains, with l VAS for overall health outcome.

The HOS is subdivided into 2 domains: HOS-ADL and HOS-Sports. The HOS-ADL is based on 17 questions, graded from 1 to 4 points, with a minimum of 17 points and a maximum of 68 points. The HOS-ADL is calculated as a percentage of the maximum of 68 points. The higher the score, the better the outcome. The minimal clinically important difference (MCID) is a common tool used to determine the smallest change in a treatment outcome that a patient would benefit from and identify as important. The MCID of the HOS-ADL at 12 
months is 23 , as reported by Chahal et al. ${ }^{20}$; they also concluded that an HOS-ADL of 80 or greater can be considered a good outcome score.

\section{Surgical Technique}

Patients were operated on in the supine position under general anesthesia. A traction table was used for subluxation of the hip joint, with fluoroscopic guidance. Two to three portals were inserted into the hip joint to adequately visualize and inspect the central compartment and the peripheral compartment for pathology, as described by Bond et al. ${ }^{21}$ Labral tears, focal chondropathy, loose bodies, pincer morphology, and peripheral cam morphology were identified and treated accordingly. Labral tears were repaired if possible or debrided otherwise. Cam and/or pincer morphologies were resected until impingement seemed resolved in flexion and with rotation of the hip joint using fluoroscopy. Focal chondropathy was treated with microfracture if suitable.

\section{Statistical Analyses}

We used IBM SPSS Statistics (version 21.0; IBM, Armonk, NY) for the descriptive statistics of our overall sample, for data cleaning and analysis, and for analyzing differences between the final subgroups. To analyze whether subgroups could be distinguished in our cohort, on the basis of the trajectories of HOS-ADL outcomes, we used Mplus $^{22}$ (version 8.1; Muthén \& Muthén, Los Angeles, CA) to perform 1-class to 6-class LCGM analyses in the form of latent class growth analysis and GMM. For all models, we specified a latent basis model for the growth pattern; the first measurement (preoperatively) and last measurement (24 months postoperatively) were fixed to 0 and 1 , respectively, and the second and third measurements ( 3 and 12 months postoperatively, respectively) were estimated freely. These estimated average slopes in our models represent the amount of change between the first and last measurements. In addition, the estimated factor loading of the second measurement explains how much of that change occurred at the 3-month (thus, the second) measurement and 12-month (thus, the third) measurement. The latent class models were independent from other variables. We based our models on a combination of visual inspection of the plots, interpretability and clinical meaningfulness of the model, and the following relative fit statistics: Bayesian information criterion, Akaike information criterion, adjusted Bayesian information criterion (in which lower values indicate a better fit), and entropy (in which higher values indicate higher confidence in the correct classification of individuals). ${ }^{14}$

A univariable analysis was performed with the R3STEP procedure in Mplus. We chose the largest subgroup of patients a priori as the reference category.
Differences between the 2 groups were compared for several factors using descriptive statistics (independent $t$ test, Student $t$ test, and $\chi^{2}$ test). The tested factors were based on the literature. ${ }^{6-8}$

\section{Predictors}

On the basis of the aforementioned literature ${ }^{6-8}$ regarding risk factors, the following potential predictors were considered: age (subdivided into $<30,30-50$, and $>50$ years), sex, BMI (subdivided into $25-30$ and $>30$ ), American Society of Anesthesiologists classification, years of pain, preoperative Tönnis grade (radiographic findings of osteoarthritis), baseline PRO scores (VAS score for pain at rest and pain with activity and HOS), and intraoperative hip pathology (cartilage damage of acetabulum or femoral head, labral tear, pincer morphology, and cam morphology). The predictors were either continuous (age, years of pain, BMI, and PRO outcomes), dichotomous (sex), or categorical (operation indication, Tönnis grade, and intraoperative pathology) and were used as such.

\section{Results}

\section{Patient Characteristics}

From 2015 through 2018, 190 patients received a diagnosis of FAI and were selected for surgery. Revision arthroscopy was performed in 22 patients, who were therefore excluded. Thus, 168 patients were eligible for participation. Of these patients, 14 were lost to follow-up, which resulted in a total of 154 patients being included in this study. Patient characteristics are presented in Table 1. Because of missing data for 1 or more HOS measurement

Table 1. Patient Characteristics

\begin{tabular}{ll}
\hline \multicolumn{1}{c}{ Characteristic } & Data $(\mathrm{N}=100)$ \\
\hline Sex & $60(60)$ \\
Female & $40(40)$ \\
$\quad$ Male & $39.2(10.9)[17-63]$ \\
Age, mean (SD) [range], yr & $25.5(3.5)[18-35]$ \\
BMI, mean (SD) [range] & \\
ASA class & $71(71)$ \\
1 & $29(29)$ \\
2 & $3.0(3.4)[0-19]$ \\
Years of pain, mean (SD) [range] & $44(44)$ \\
Preoperative Tönnis grade & $45(45)$ \\
0 & $11(11)$ \\
1 & \\
2 & $52(52)$ \\
Intraoperative hip pathology & $22(22)$ \\
Cam & $83(83)$ \\
Pincer & $30(30)$ \\
Labral tear & $42(42)$ \\
Cartilage damage of head &
\end{tabular}

NOTE. Data are presented as number (percentage) unless otherwise indicated.

ASA, American Society of Anesthesiologists; BMI, body mass index; $\mathrm{SD}$, standard deviation. 
Table 2. VAS Pain Score, HOS-Sports, and HOS-ADL at Baseline and During Follow-up

\begin{tabular}{|c|c|c|c|c|}
\hline & Baseline & 1- yr Follow-up & 2-yr Follow-up & $P$ Value \\
\hline$\overline{\text { VAS pain score }(0-100)}$ & $63.1(23.8)$ & $13.2(1.6)$ & NA & $<.001$ \\
\hline HOS-Sports & $51.1(24.4)$ & $76.6(24.11)$ & $73.7(24.05)$ & $<.006$ \\
\hline HOS-ADL & $65.7(20.1)$ & $88.2(13.8)$ & $86.0(14.3)$ & $<.001$ \\
\hline
\end{tabular}

NOTE. Data are presented as mean (standard deviation).

HOS, Hip Outcome Score; HOS-ADL, Hip Outcome Score-Activities of Daily Life; NA, not asked; VAS, visual analog scale.

*Significance level set at $P<.05$ using paired $t$ test.

questions for several patients, data for analysis in Mplus were complete for 100 patients in total. Descriptive statistics for the PRO scores (VAS pain score, HOS-Sports, and HOS-ADL) are presented in Table 2.

\section{Selection of Best Model}

We based our models on several model fit statistics, presented in Table 3. On the basis of these criteria, we chose the 2-class GMM as our final model in recovery trajectory. Although the fit statistics continued to decrease up to the 6-class model, this decrease already started to flatten out from the 2-class model and upward (Fig 1). This finding indicates that from the 3-class model and further upward, the new classes did not increase the clinical meaningfulness of the model because they were mostly slight variations of the classes from the 2-class model. All GMMs showed the same type of trajectory: one fairly homogeneous class with a good improving model for functional recovery, as well as one or more other models with only minor improvement, no improvement, or even a minor decrease in functional outcome, with a wide range of outcome results. These minor improvements or decreases in outcome score (HOS-ADL) were all small and below the MCID of 23 points. ${ }^{20}$ The smaller classes became smaller and more heterogeneous from the 3-class models upward, thereby limiting its clinical meaningfulness. In addition, from the 4-class model upward, errors occurred in analysis owing to local maxima, indicating the possibility that the results from those models may not be trustworthy. The estimations of the factor loadings (i.e., the percentages at 3 and 12 months of the total change) are therefore unreliable; these models were not suitable for our data (Table 4). Adding more classes to the 2-class model did not improve the model; therefore, we chose the 2-class model, as shown in Fig 1.

\section{Trajectory Patterns}

We labeled the first class as the "improvers" because this class - the largest (78 patients) - was portrayed by a steep improvement in HOS-ADL values during the first 3 months, starting at a baseline score of 68.5; the HOSADL values subsequently leveled out after 1-year follow-up. At 24 months, the improvers reached 92.2 on the HOS-ADL, which is defined as a good result. ${ }^{20}$

A more detailed figure of the first class showed a wide range mainly in the start at baseline. All patterns showed the same type of positive recovery during follow-up (Fig 2).

The second class consisted of 22 patients and was labeled as the "non-improvers." This class showed a minor improvement in HOS-ADL values, lower than the MCID of 23, starting from a mean estimated baseline score of 62.6 and ending with a mean estimated score of 65.5. The largest part of this improvement occurred during the first 3 months after surgery. During follow-up, there was only minor improvement in the HOS-ADL at 2 years compared with preoperative scores: a 3-point improvement to a score of 62, which is a non-clinically relevant difference (below the MCID) and less than $80 \%$ of the total HOS-ADL value, which was defined by Chahal et al. ${ }^{20}$

The second class showed a wider range in recovery patterns, which is projected in Fig 3. Figs 2 and 3 demonstrate that some observed individual values are not a perfect fit to the estimated trajectory within its class. However, homogeneity within the classes did not improve when adding classes to the model, as described in the "Methods" section.

Table 3. Summary of Model Fit Statistics

\begin{tabular}{|c|c|c|c|c|c|c|c|}
\hline Model: GMM & LL & BIC & AIC & Adjusted BIC & BLRT & Entropy & No. of Free Parameters \\
\hline$\overline{1 \text { Class }}$ & $-1,599.779$ & $3,250.215$ & $3,221.558$ & $3,215.474$ & - & - & 11 \\
\hline 2 Classes & $-1,567.497$ & $3,208.677$ & $3,166.994$ & $3,158.145$ & - & 0.87 & 16 \\
\hline 3 Classes & $-1,546.976$ & $3,190.66$ & $3,135.951$ & $3,124.336$ & - & 0.878 & 21 \\
\hline 5 Classes & $-1,532.085$ & $3,206.93$ & $3,126.17$ & $3,109.025$ & - & 0.867 & 31 \\
\hline 6 Classes & $-1,519.216$ & $3,204.218$ & $3,110.432$ & $3,090.521$ & - & 0.914 & 36 \\
\hline
\end{tabular}

AIC, Akaike information criterion; BIC, Bayesian information criterion; BLRT, bootstrapped likelihood ratio test; GMM, growth mixture modeling; LL, log likelihood. 


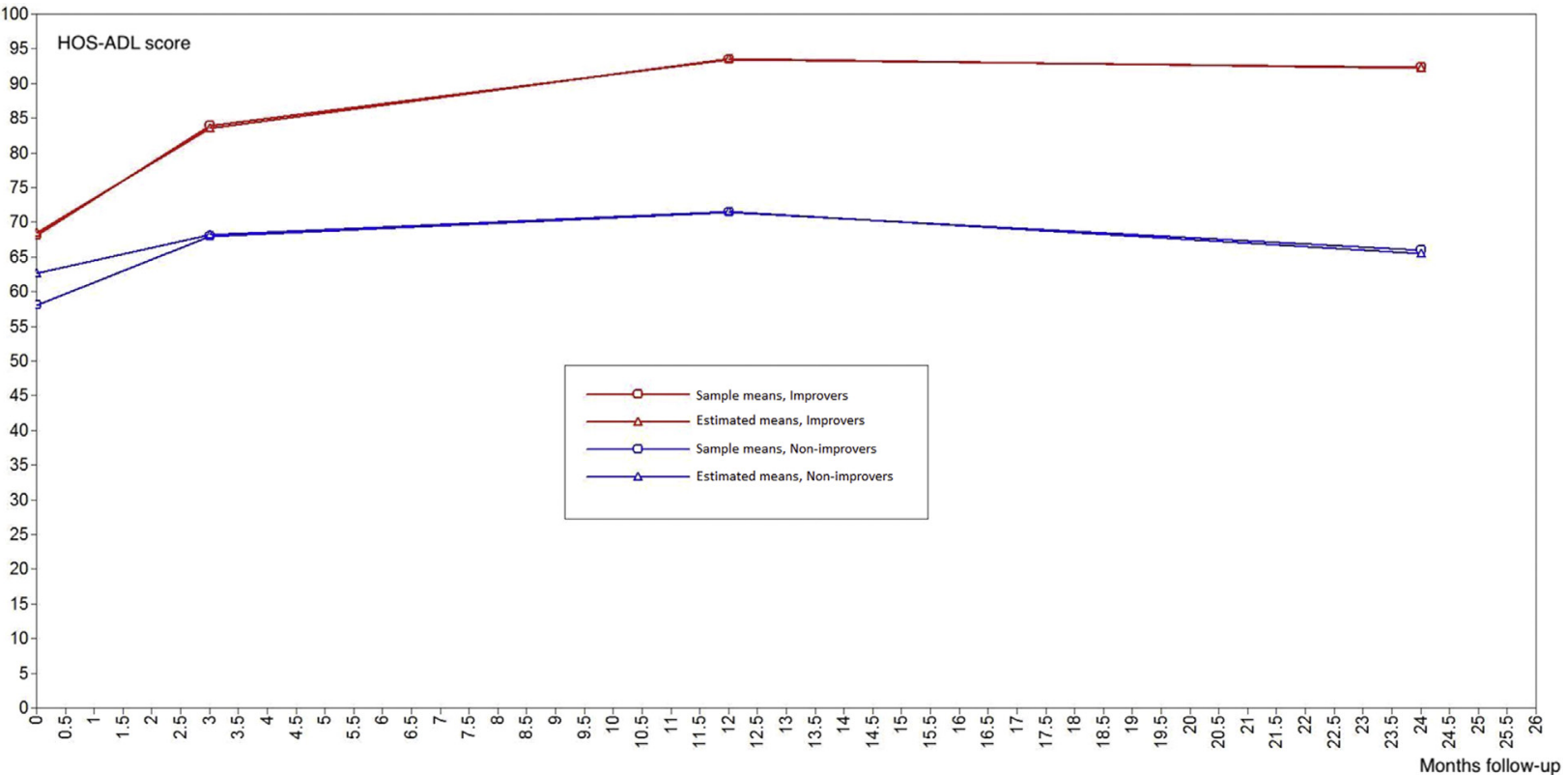

Figure 1. Estimated means and sample means of selected 2-class model, which were named improvers and non-improvers for recovery trajectory: 1 good recovering class and 1 fairly poor recovering class. (HOS-ADL, Hip Outcome Score-Activities of Daily Life.)

Class Membership Based on Patient Characteristics

The overall patient characteristics are presented in Table 1. Univariable analysis and comparisons of means and differences in several factors were used to identify differences between classes. The improver class was chosen as the reference category.

Table 4. Model Parameter Factor Loading at 3 and 12 Months, Intercept, Slope, and Class Size

\begin{tabular}{|c|c|c|c|c|c|}
\hline Model and Class & $\begin{array}{c}\text { Factor Loading of } \\
\text { HOS at } 3 \text { mo }\end{array}$ & $\begin{array}{c}\text { Factor Loading of } \\
\text { HOS at } 12 \mathrm{mo}\end{array}$ & Intercept (SE) & Slope (SE) & $\begin{array}{l}\text { Patients } \\
\text { per Class }\end{array}$ \\
\hline$\overline{1-C l a s s ~ G M M}$ & $0.719(0.062)$ & $1.115(0.055)$ & $65.642(1.998)$ & $20.263(2.028)$ & 100 \\
\hline \multicolumn{6}{|l|}{ 2-Class GMM } \\
\hline Class 2 & $1.917(1.099)$ & $3.092(1,754)$ & $62.575(7.005)$ & $2.902(2,977)$ & 22 \\
\hline \multicolumn{6}{|l|}{ 3-Class GMM } \\
\hline Class 1 & $0.740(0.078)$ & $1.43(0.032)$ & $71.599(3,143)$ & $25,147(3,236)$ & 46 \\
\hline \multicolumn{6}{|l|}{ 4-Class GMM } \\
\hline Class 1 & $0.815(0.182)$ & $0.986(0.032)$ & $71.910(2.886)$ & $26.604(3.047)$ & 49 \\
\hline Class 2 & $1.962(0.643)$ & $2.250(0.457)$ & $38.212(5.277)$ & $10.934(6.483)$ & 5 \\
\hline Class 3 & $0.393(0.182)$ & $1.238(0.114)$ & $62.510(5.945)$ & $20.506(6.995)$ & 33 \\
\hline Class 4 & $0.472(0.247)$ & $-0.051(0.167)$ & $68.485(3.452)$ & $0.003(5.064)$ & 13 \\
\hline \multicolumn{6}{|l|}{ 5-Class GMM } \\
\hline Class 5 & $0.723(0.256)$ & $-0.063(0.261)$ & $73.543(4.533)$ & $9.334(4.461)$ & 10 \\
\hline \multicolumn{6}{|l|}{ 6-Class GMM } \\
\hline Class 1 & $2.261(0.690)$ & $2.939(0.567)$ & $39.598(4.819)$ & $8.797(6.075)$ & 5 \\
\hline Class 2 & $0.699(0.106)$ & $1.038(0.031)$ & $70.703(2.781)$ & $26.178(2.763)$ & 47 \\
\hline Class 3 & $0.516(0.231)$ & $1.419(0.231)$ & $63.812(7.139)$ & $16.561(6.695)$ & 22 \\
\hline Class 4 & $*$ & $*$ & * & $*$ & 0 \\
\hline Class 5 & $0.506(0.318)$ & $-0.263(0.212)$ & $68.626(2.535)$ & $-2.125(3.275)$ & 13 \\
\hline Class 6 & $0.535(0.208)$ & $0.307(0.240)$ & $79.895(3.097)$ & $11.010(4.346)$ & 13 \\
\hline
\end{tabular}

GMM, growth mixture modeling; HOS, Hip Outcome Score; SE, standard error.

*Owing to an error in the calculations, the best log-likelihood value was not replicated. The solution may not be trustworthy because of local maxima, and 1 or more parameters were fixed to avoid singularity of the information matrix. 


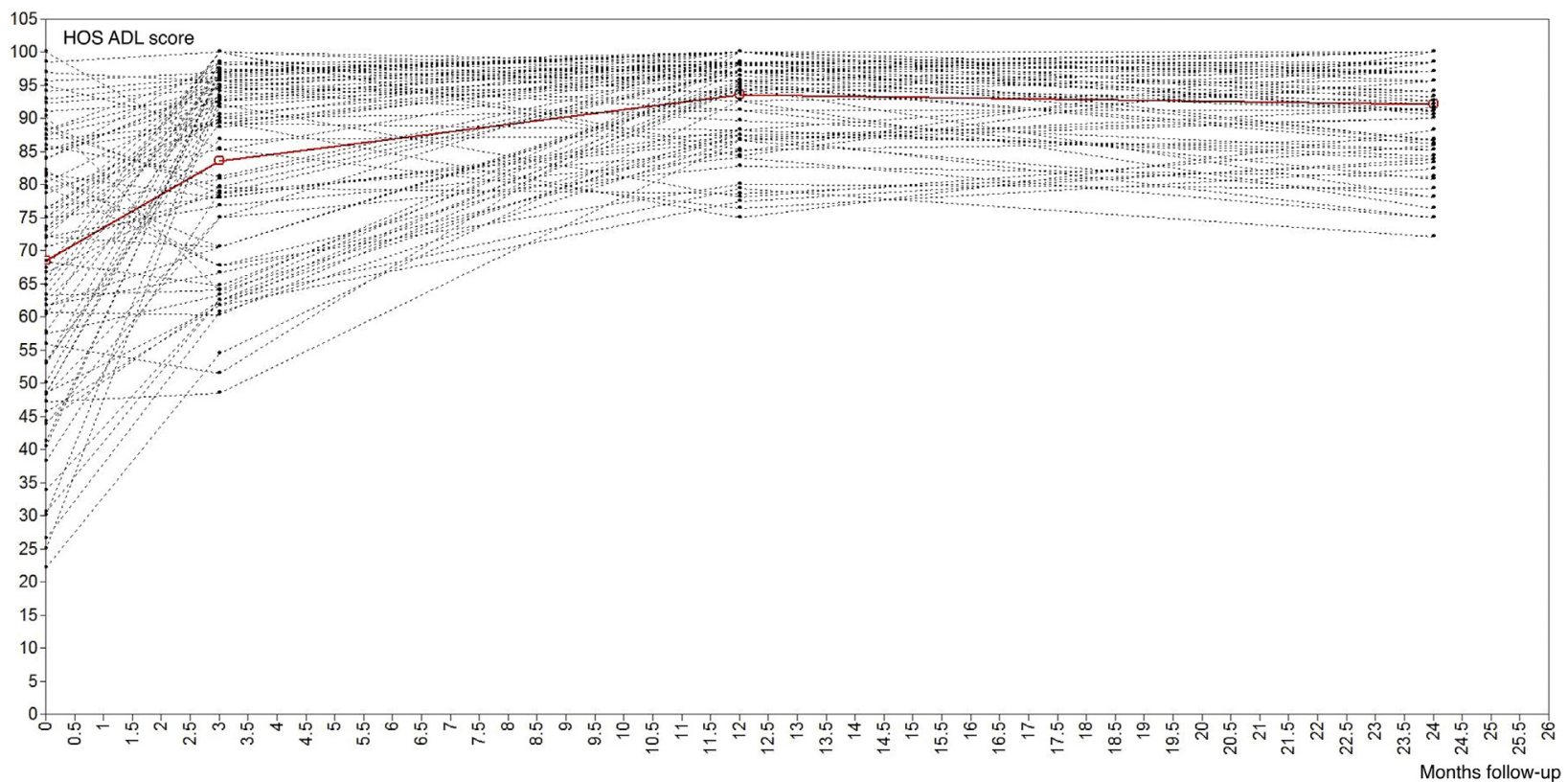

Figure 2. Observed individual values and estimated means for class 1: improvers. (HOS-ADL, Hip Outcome Score-Activities of Daily Life.)

In the univariable analysis (Table 5), the following variables were statistically significant for membership in the non-improver class: high VAS pain score preoperatively $(P=.007)$ and intraoperative arthrosis of the femoral head $(P=.025)$. We compared means and differences in several factors of both groups as mentioned in the "Methods" section using descriptive statistics. The results are presented in Table 6.

\section{Discussion}

We identified 2 main subgroups with different functional recovery trajectories in our sample of 154 patients operated on for FAI syndrome with hip arthroscopy. We named the subgroups the improvers and the non-improvers. On the basis of our results using the HOS-ADL as an outcome measure, the improvers can be seen as having an ideal recovery

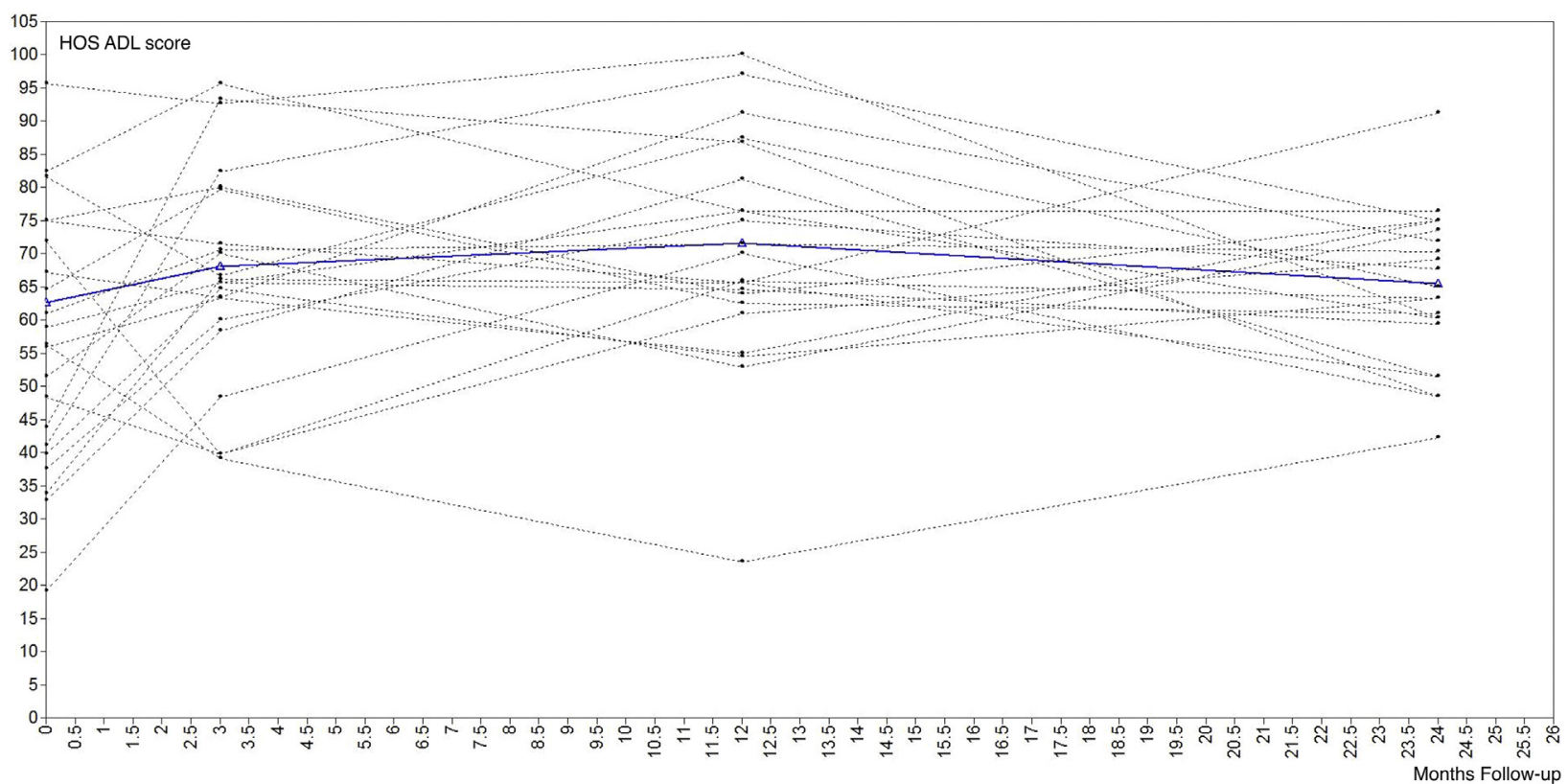

Figure 3. Observed individual values and estimated means for class 2: non-improvers. (HOS-ADL, Hip Outcome Score-Activities of Daily Life.) 
Table 5. Outcomes of Univariable Analysis With Non-improver Class Membership as Dependent Variable

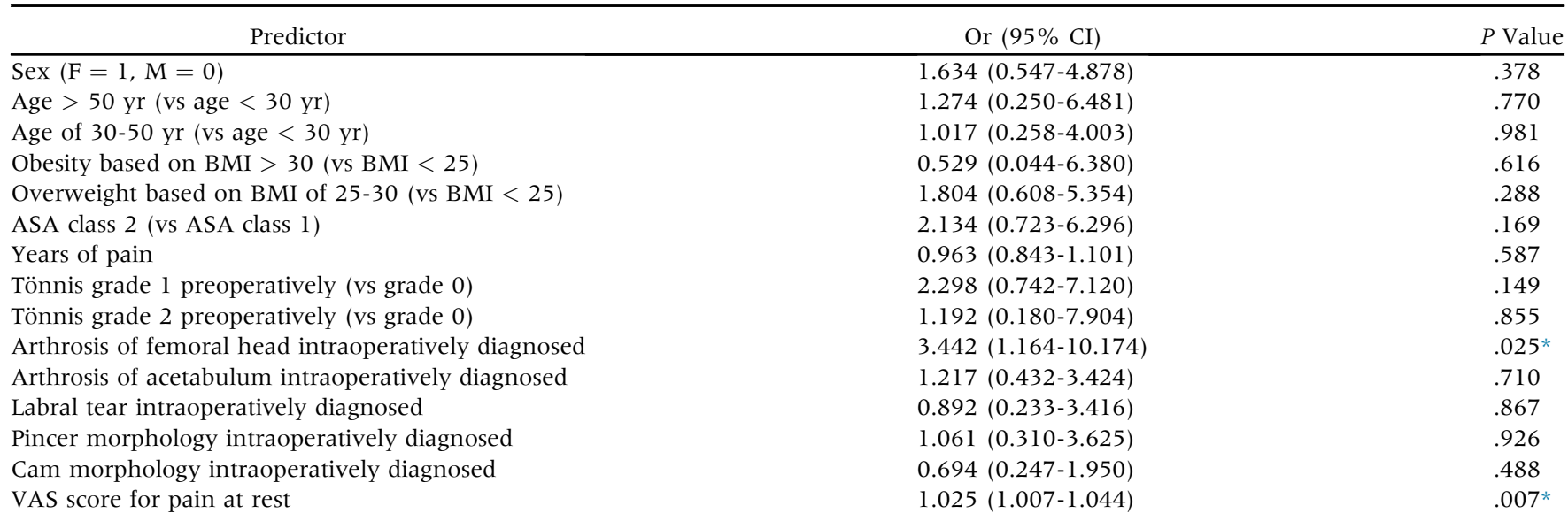

ASA, American Society of Anesthesiologists; BMI, body mass index; CI, confidence interval; F, female; M, male; OR, odds ratio; VAS, visual analog scale.

*Statistically significant $(P<.05)$.

trajectory for FAI syndrome patients. The nonimprovers can be seen as having a less favorable trajectory because postoperative recovery is lower in this class. By use of univariable analysis and descriptive statistics, differences between the improvers and nonimprovers were found in the preoperative VAS score for pain at rest and for intraoperative cartilage damage of the femoral head. Multivariable analysis cannot be performed because our cohort is too small to be able to draw definitive conclusions from such an analysis.
Other studies have identified certain variables with varying effects on functional outcomes after FAI syndrome surgery, such as female sex, higher age, labral tears, pincer morphology, cartilage degeneration, chondral defects, acetabular coverage (high lateral centeredge angle), and femoral pistol-grip deformity. ${ }^{7,23-26}$ We could not define the true influence of any of these variables on class membership because multivariable analysis was not feasible. In a larger cohort, such an analysis is feasible. For example, Hesseling et al. ${ }^{9}$ used

Table 6. Differences Between Improver and Non-improvers Subgroups

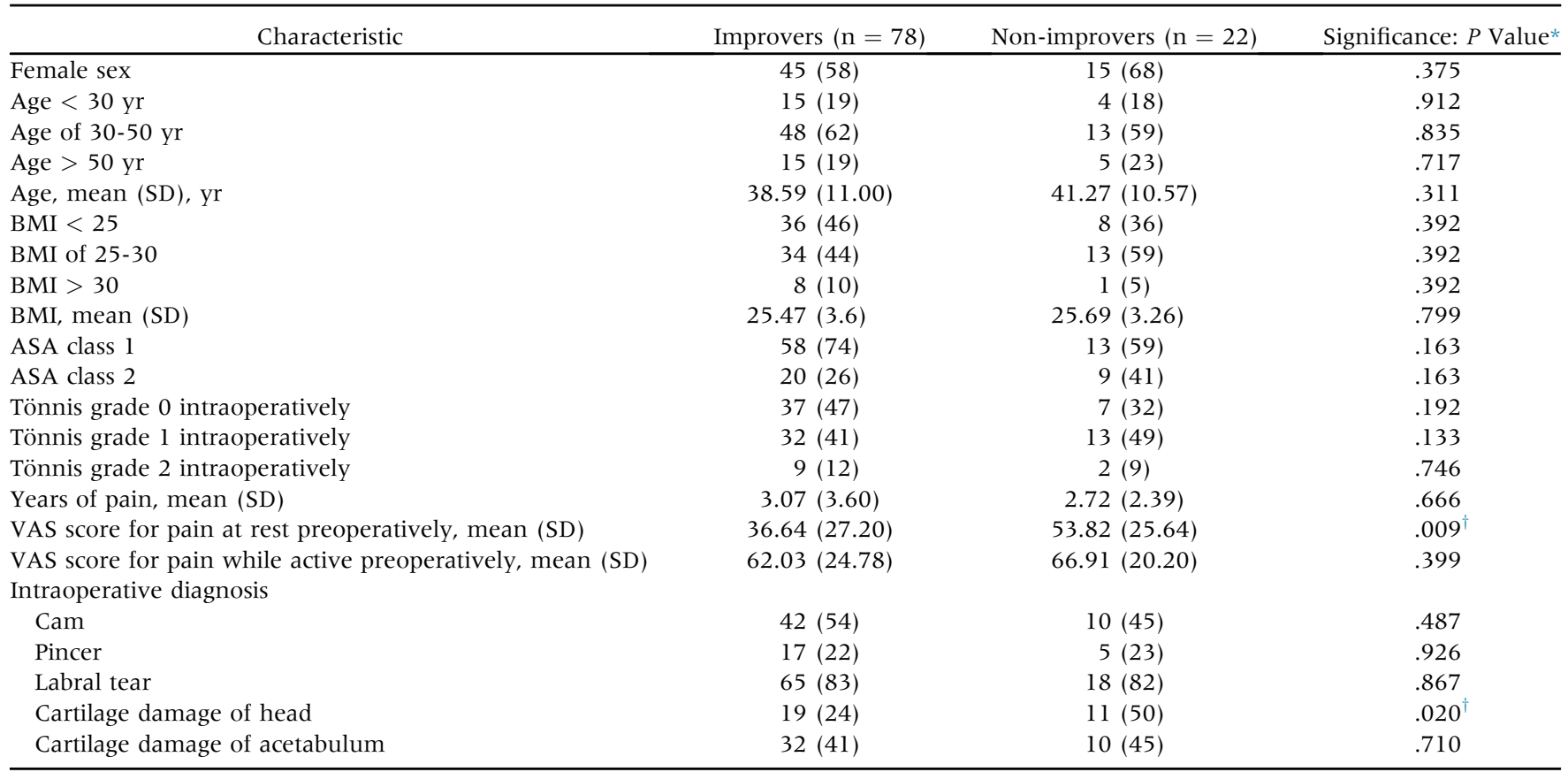

NOTE. Data are presented as number (percentage) unless otherwise indicated.

ASA, American Society of Anesthesiologists; BMI, body mass index; SD, standard deviation; VAS, visual analog scale.

*Tested with independent $t$ test and $\chi^{2}$ test.

${ }^{\dagger}$ Statistically significant $(P<.05)$. 
LCGM to study a cohort of over 6,000 patients and found multiple predictors of functional recovery trajectories after total hip arthroplasty.

An important finding of our study is that the GMM identifies subgroups of patient recovery patterns after arthroscopic surgery for FAI. This is a type of postoperative functional outcome analysis, which identifies subgroups in their recovery. This study identified 1 clearly superior recovery model. Unfortunately, this data set did not include enough patients to properly identify risk factors for group membership with a multivariable regression analysis. Larger cohort studies must be analyzed with LCGM or GMM to identify covariates for class membership. This future research might be able to improve the understanding of how class membership differs and whether patients can preoperatively be "upgraded" to be able to join the favorable class.

Arthroscopic surgery for FAI syndrome can significantly resolve pain and impairment in patients. ${ }^{1,27,28}$ However, not all patients benefit equally from surgery. Careful patient selection for surgery is important to obtain good results in patients in terms of functional recovery and pain relief. We identified differences in intraoperative cartilage damage of the femoral head and the preoperative pain score between the groups. This finding is understandable: More cartilage damage of the femoral head causes higher levels of preoperative pain in patients and cannot be repaired with surgery, therefore causing more pain afterward with poorer outcomes in terms of functional results. This GMM study confirms that cartilage damage is a risk factor for poor recovery and shows the difference in recovery pattern.

Our study can be seen as an exploratory study and is an important step in achieving a better understanding of the amount of heterogeneity in FAI patients' recovery after hip arthroscopy. The outcome can be used a guidance tool in patient selection and counseling of patients regarding their expectation management for recovery after surgery. It is known that preoperative expectations can affect satisfaction after surgery. ${ }^{29}$ Parameters that can help to predict the outcome of surgery are helpful in preoperative expectation management. This, in turn, might help to improve the outcome of surgery. For example, when patients have severe preoperative pain, represented by a high VAS score for pain at rest preoperatively, it can be helpful to counsel patients that persisting symptoms of pain can be maintained postoperatively.

We identified 2 different subgroups based on different trajectory outcomes, which were based on the functional improvement postoperatively measured with the HOSADL. A significant improvement in the HOS-ADL is defined as a total score greater than 80 or as improvement that exceeds the MCID defined by Chahal et al., ${ }^{20}$ which is 23 at 12 months. The MCID is a common tool used to determine the smallest change in a treatment outcome that a patient would benefit from and identify as important. The MCID of the HOS-ADL was calculated at 23, as reported by Chahal et al. Other authors, however, have used MCIDs of 8.3 and 9 points for the HOS-ADL (with a total of 68 points). ${ }^{30}$ It is not entirely clear to us how these different MCID values can be explained, besides pointing out differences in the cohort size ( 130 patients in the cohort of Chahal et al. and 364 patients in that of Nwachukwu et al. ${ }^{30}$ ) and the timing of MCID calculations (calculated at 3, 6, and 12 months by Chahal et al. and at 12 months by Nwachukwu et al.).

The favorable recovery pattern (improvers) had a recovery in HOS-ADL values to over 90. The improvement from 68.5 to 92.2 is slightly higher than the MCID of 23 for the HOS-ADL as defined by Chahal et al. ${ }^{20}$ and is much larger than the MCIDs of 8.3 and 9 points as defined by Nwachukwu et al. ${ }^{30}$ and Martin and Philippon. ${ }^{31}$

Another parameter for measuring successful recovery is the VAS score for pain. The overall improvement in VAS pain scores in our cohort was from 4.1 to 1.7, which is a decrease of 2.4. The MCID for the VAS pain score was calculated as $1.48 .^{32}$ Overall, the improver trajectory can be considered a successful recovery.

Another point of discussion in our study is that we used the HOS-ADL as the primary outcome. Several PRO questionnaires are available and can be used to measure functional outcomes after hip arthroscopy. These PRO questionnaires were designed and used to measure end scores for recovery after surgery. We tried to identify trajectories of recovery and not only the endpoints. In this study, we had to choose 1 specific questionnaire. Another questionnaire that could have been used is the International Hip Outcome Tool 12 (iHOT-12). We did not include the iHOT-12 questionnaire from the beginning of our registration; therefore, we did not have complete data for this questionnaire. The iHOT-12 questionnaire is now, however, included in our daily practice.

A strength of this study is the unique analysis of recovery trajectories, thereby providing a more detailed understanding of the degree of variation between patients in recovery after hip arthroscopy for FAI syndrome. We used data from all patients, including those with poor outcomes after surgery.

\section{Limitations}

Our study is not without limitations. One limitation is our rate of loss to follow-up: Of 168 initial patients suitable for study participation, 14 were lost to followup. The percentage lost to follow-up is acceptable but given that a less than $5 \%$ rate of loss to follow-up can lead to a small amount of bias, ${ }^{33}$ this must be taken into 
account in our study. In addition, in our study, many patients had incomplete questionnaires during followup. A total of 54 patients had incomplete questionnaires, which could not be handled other than by exclusion from our data analysis. Because of this incompleteness of our data, a total of 100 patients were analyzed. We consider this a clear limitation in our data. Another limitation is that our sample size was not large enough to perform a multivariable regression analysis to truly identify risk factors for group membership.

\section{Conclusions}

We identified 2 main types of recovery pattern after arthroscopic treatment of FAI syndrome: improvers and non-improvers. Both groups recover in a different manner postoperatively. Preoperative pain and intraoperative arthrosis of the femoral head differed in the non-improvers compared with the improvers. This study is an exploratory study; these results and future research can help surgeons in providing better preoperative consultations and expectation management.

\section{References}

1. Levy DM, Kuhns BD, Chahal J, Philippon MJ, Kelly BT, Nho SJ. Hip arthroscopy outcomes with respect to patient acceptable symptomatic state and minimal clinically important difference. Arthroscopy 2016;32:1877-1886.

2. Kierkegaard S, Langeskov-Christensen M, Lund B, et al. Pain, activities of daily living and sport function at different time points after hip arthroscopy in patients with femoroacetabular impingement: A systematic review with meta-analysis. Br J Sports Med 2017;51:572-579.

3. Khan M, Habib A, de Sa D, et al. Up to date: Hip femoroacetabular impingement. Arthroscopy 2016;32:177-189.

4. Menge TJ, Briggs KK, Dornan GJ, McNamara SC, Philippon MJ. Survivorship and outcomes 10 years following hip arthroscopy for femoroacetabular impingement: Labral debridement compared with labral repair. J Bone Joint Surg Am 2017;99:997-1004.

5. Griffin DR, Dickenson EJ, O'Donnell J, et al. The Warwick Agreement on femoroacetabular impingement syndrome (FAI syndrome): An international consensus statement. Br J Sports Med 2016;50:1169-1176.

6. Sardana V, Philippon MJ, de Sa D, et al. Revision hip arthroscopy indications and outcomes: A systematic review. Arthroscopy 2015;31:2047-2055.

7. Saadat E, Martin SD, Thornhill TS, Brownlee SA, Losina E, Katz JN. Factors associated with the failure of surgical treatment for femoroacetabular impingement: Review of the literature. Am J Sports Med 2014;42: 1487-1495.

8. Sochacki KR, Brown L, Cenkus K, Di Stasi S, Harris JD, Ellis TJ. Preoperative depression is negatively associated with function and predicts poorer outcomes after hip arthroscopy for femoroacetabular impingement. Arthroscopy 2018;34:2368-2374.

9. Hesseling B, Mathijssen NMC, van Steenbergen LS, Melles M, Vehmeijer SBW, Porsius JT. Fast starters, slow starters and later dippers: Trajectories of patient-reported outcomes after total hip arthroplasty: Results from a Dutch nationwide database. J Bone Joint Surg Am 2019;101:2175-2186.

10. Bauer DJ. Estimating multilevel linear models as structural equation models. J Educ Behav Stat 2003;28:135-167.

11. Laird NM, Ware JH. Random-effects models for longitudinal data. Biometrics 1982;38:963-974.

12. Berlin KS, Parra GR, Williams NA. An introduction to latent variable mixture modeling (part 2): Longitudinal latent class growth analysis and growth mixture models. J Pediatr Psychol 2014;39:188-203.

13. Ram N, Grimm K. Using simple and complex growth models to articulate developmental change: Matching theory to method. Int J Behav Dev 2007;31:303-316.

14. Ram N, Grimm KJ. Growth mixture modeling: A method for identifying differences in longitudinal change among unobserved groups. Int J Behav Dev 2009;33:565-576.

15. Porsius JT, Mathijssen NM, Klapwijk-Van Heijningen LC, Van Egmond JC, Melles M, Vehmeijer SB. Early recovery trajectories after fast-track primary total hip arthroplasty: The role of patient characteristics. Acta Orthop 2018;89: 597-602.

16. Egmond JC, Hesseling B, Melles M, et al. Three distinct recovery patterns following primary total knee arthroplasty: Dutch arthroplasty register study of 809 patients. Knee Surg Sport Traumatol Arthrosc 2021;29:529-539.

17. Arthur HM, Blanchard C, Gunn E, Kodis J, Walker S, Toner B. Exercise trajectories of women from entry to a 6-month cardiac rehabilitation program to one year after discharge. Biomed Res Int 2013;2013:121030.

18. Martin HD, Kelly BT, Leunig M, et al. The pattern and technique in the clinical evaluation of the adult hip: The common physical examination tests of hip. Arthroscopy 2010;26:161-172.

19. Rabin R, Gudex C, Selai C, Herman M. From translation to version management: A history and review of methods for the cultural adaptation of the EuroQol fivedimensional questionnaire. Value Health 2014;17:70-76.

20. Chahal J, van Thiel GS, Mather RC, Lee S, Salata MJ, Nho SJ. The minimal clinical important difference (MCID) and patient acceptable symptomatic state (PASS) for the modified Harris Hip Score and Hip Outcome Score among patients undergoing surgical treatment for femoroacetabular impingement. Orthop $J$ Sports Med 2014;2(suppl):2325967114S00105.

21. Bond JL, Knutson ZA, Ebert A, Guanche CA. The 23-point arthroscopic examination of the hip: Basic setup, portal placement, and surgical technique. Arthroscopy 2009;25: 416-429.

22. Muthén LK, Muthén BO. MPlus user's guide, Ed 8. Los Angeles: Muthén \& Muthén, 2017.

23. Haefeli PC, Albers CE, Steppacher SD, Tannast M, Büchler L. What are the risk factors for revision surgery after hip arthroscopy for femoroacetabular impingement at 7-year followup? Clin Orthop Relat Res 2017;475: 1169-1177.

24. Stephan P, Röling MA, Mathijssen NMC, Hannink G, Bloem RM. Developing a risk prediction model for the functional outcome after hip arthroscopy. BMC Musculoskelet Disord 2018;19:122. 
25. Egerton T, Hinman RS, Takla A, Bennek KL, O’Donnell J. Intraoperative cartilage degeneration predicts outcome 12 months after hip arthroscopy. Clin Orthop Relat Res 2013;471:593-599.

26. Sogbein OA, Shah A, Kay J, et al. Predictors of outcomes after hip arthroscopic surgery for femoroacetabular impingement: A systematic review. Orthop J Sports Med 2019;19(7):2325967119848982.

27. Kaldau NC, Brorson S, Hölmich P, Lund B. Good midterm result of hip arthroscopy for femoroacetabular impingement. Dan Med J 2018;65:A5483.

28. Philippon MJ, Stubbs AJ, Schenker ML, Maxwell RB, Ganz R, Leuning M. Arthroscopic management of femoroacetabular impingement: Osteoplasty technique and literature review. Am J Sports Med 2008;35:1571-1580.

29. Swarup I, Henn CM, Gulotta LV, Henn RF. Patient expectations and satisfaction in orthopaedic surgery: A review of the literature. J Clin Orthop Trauma 2019;10: 755-760.

30. Nwachukwu BU, Fields K, Chang B, Nawabi DH, Kelly BT, Ranawat AS. Preoperative outcome scores are predictive of achieving the minimal clinically important difference after arthroscopic treatment of femoroacetabular impingement. Am J Sports Med 2017;45:612-619.

31. Martin RR, Philippon M. Evidence of reliability and responsiveness for the Hip Outcome Score. Arthroscopy 2008;24:676-682.

32. Beck EC, Mwachukwu BU, Kunze KN, Chahla J, Nho SJ. How can we define clinically important improvement in pain scores after hip arthroscopy for femoroacetabular impingement syndrome? Minimum 2-year follow-up study. Am J Sports Med 2019;47:3133-3140.

33. Dettori JR. Loss to follow-up. Evid Based Spine Care J 2011;2:7-10. 